\title{
LEER A MAQUIAVELO, TRADUCIR EL CORÁN: MUḤAMMAD, PRÍNCIPE Y LEGISLADOR EN EL ALCORANO DI MACOMETTO (VENECIA, 1547) *
}

\author{
READING MACHIAVELLI, TRANSLATING THE \\ QUR'ĀN: MUHAMMAD AS A LAWGIVER IN THE \\ ALCORANO DI MACOMETTO (VENICE, 1547)
}

\author{
Pier Mattia Tommasino \\ CCHS-CSIC, Madrid, España
}

\begin{abstract}
Este artículo se centra en L'Alcorano di Macometto, escrito por Giovanni Battista Castrodardo de Belluno (1517 ca.-1588 ca.) e impreso por Andrea Arrivabene en Venecia en 1547. Aunque hoy sea considerado como una torpe paráfrasis de la traducción latina del Corán de Roberto de Ketton (1143 AD), un examen atento demuestra que L'Alcorano costituyó una práctica y exitosa enciclopedia de bolsillo sobre la historia del islam y del Imperio otomano. Siguiendo una intuición de Alessandro D'Ancona (1889), analizo los textos históricos publicados en la introducción del texto, centrándome especialmente en la biografía del profeta Muhammad escrita por Castrodardo. Dicha biografía presenta una larga oración renacentista pronunciada por el monje Sergio-Bahīrā para convencer a $\mathrm{Mu}$ hammad a derrocar al emperador Heraclio.
\end{abstract}

This article focuses on L'Alcorano di Macometto, written by Giovanni Battista Castrodardo from Belluno (1588 ca.-1517 ca.), and published by Andrea Arrivabene at Venice in 1547. Although this text is considered to be a paraphrase of the Latin translation of the Qur'ān made by Robert of Ketton (1143 AD), a closer look shows that it was a handy pocket encyclopedia of the history of Islam and the Ottoman empire. Following a suggestion of Alessandro D'Ancona (1889), I analyze the historical texts published in the introduction to the Alcorano and especially Castrodardo's biography of the prophet $\mathrm{Mu}-$ hammad. This biography includes a prayer addressed to Muhammad through the character of the monk Sergio-Bahīrā in order to persuade him to dethrone Emperor Heraclius. A deep linguistic analysis of this speech

* Este ensayo ha sido presentado en forma de comunicación en el Instituto de Lenguas y Culturas del Mediterráneo y Oriente Próximo del Centro de Ciencias Humanas y Sociales (CCHS,CSIC) de Madrid el 7 de junio 2011, gracias a la financiación del Lila Wallace-Reader's Digest Endowment Fund at Villa I Tatti (Harvard, Florence). El texto utilizado es L'Alcorano di Macometto, nel qual si contiene la dottrina, la vita, $i$ costumi, et le leggi sue. Tradotto nuovamente dall'Arabo in lingua italiana. Con Gratie e Privilegii. [Venezia, Andrea Arrivabene] MDXLVII, Roma, Biblioteca Angelica, H.6.20. 
A través de un análisis lingüístico y retórico detallado de esta oración, intento demostrar la cultura literaria de Castrodardo y su interés por las obras de Maquiavelo. Este nuevo retrato de Muhammad presenta al profeta del islam como un príncipe renacentista, un legislador y un «profeta armado», revelando asimismo las ideas políticas de los círculos filoturcos y anti-imperiales vinculados a la embajada de Francia en Venecia.

Palabras clave: Castrodardo; Arrivabene; Alcorano di Macometto; Muhammad; Sergio; Bahīīā; Maquiavelo; El Príncipe; legislador; Luetz d'Aramon. sheds light on Castrodardo's reading of Machiavelli. While this new portrayal of $\mathrm{Mu}$ hammad is based on medieval sources, it presents the Prophet of Islam as a distinctly Renaissance Prince, a lawgiver and a "Prophet Armed". It also reflects the pro-Ottoman and anti-Imperial hopes of Italian circles linked to the French embassy in Venice.

Key words: Castrodardo; Arrivabene; Alcorano di Macometto; Muhammad; Sergio; Bahīrā; Machiavelli; The Prince; Lawgiver; Luetz d'Aramon.

\section{L'Alcorano di Macometto, Venecia, 1547}

L'Alcorano di Macometto, texto impreso en Venecia en 1547 por el editor Andrea Arrivabene sin nombre de autor, puede ser considerado como una práctica enciclopedia de bolsillo sobre el islam en el que también figura la primera traducción impresa del Corán en una lengua nacional europea. La obra está dedicada al embajador francés en el Imperio Otomano, el barón provenzal Gabriel Luetz d'Aramon, encargado de negociar una alianza militar antiimperial entre Francia y los Otomanos (1547-1553). D'Aramon vivió en Venecia entre 1540 y 1542, período durante el cual fue amigo de Aretino y retratado por Tiziano. Después de una larga misión en Estambul, recibió por parte de Francisco I el cargo de embajador oficial permaneciendo en Venecia entre el 9 y el 24 de febrero 1547.

Descrito desde el siglo XVIII como una edición muy rara en los catálogos europeos (editio perrara, fort rare et recherchée, libro assai raro, libro rarissimo, etc.), L'Alcorano tuvo en realidad un importante éxito editorial, generalmente ignorado por los historiadores de la imprenta europea y veneciana. Su edición definitiva consta de tres libros (100 hojas), precedidos por una larga introducción (50 hojas). Tanto la introducción como el primer libro reúnen textos históricos, medievales y contemporáneos, sobre el islam y su profeta así como sobre las costumbres de los turcos, mientras que el segundo y tercer libro contienen la traducción italiana del Corán, basada en la traducción latina medieval de Roberto de Ketton (1143) impresa en Basilea en 1543 por el teólogo y orientalista Theodor Buchmann, 
conocido como Bibliander (1506-1564) y el editor Johannes Oporinus.

\section{Giovanni Battista Castrodardo, traductor, dantista, historiador}

El traductor-compilador de la obra, como ya demostré en un artículo publicado en diciembre de 2008, es sin duda Giovanni Battista Castrodardo (1517 ca.-1588 ca.), canónigo de la catedral de Belluno desde 1534 a 1584, y polígrafo desconocido para la historia de la imprenta y del Renacimiento italiano, aunque muy activo en Padua y Venecia entre 1543 y $1547^{1}$.

Castrodardo es autor, al menos, de cuatro obras. Su primer trabajo se remonta a 1544: Li tre libri di Nicolò Leonico De varie historie, es decir, la traducción italiana, impresa por Michele Tramezino, de los De varia historia libri III del humanista veneciano Niccolò Leonico Tomeo (1456 ca.-1531) publicados por Luca Antonio Giunta en $1521^{2}$.

La segunda obra permaneció manuscrita y hasta ahora está desaparecida. Se trata de un comentario sobre la Commedia de Dante, escrito entre 1544 y los primeros meses de 1547, que tuvo una profunda influencia en la composición de L'Alcorano di Macometto y en particular en la narración del episodio del mi 'rāây adaptado de la traducción italiana de la Confusión de la secta mahomética del morisco jativense y canónigo de la catedral de Granada Juan Andrés. Algunos fragmentos del comentario estuvieron en posesión de la familia Castrodardo por lo menos hasta 1720, como nos recuerda una carta enviada por el notario Aleandro Castrodardo al periodista e intelectual veneciano Apostolo Zeno (1668-1750), que al principio del siglo XVIII iba reuniendo fuentes literarias para la composición de una historia de la poesía italiana ${ }^{3}$.

1 Tommasino, "Giovanni Battista Castrodardo".

${ }^{2}$ Leonico Tomeo, Nicolai Leonici Thomaei; Leonico Tomeo, Li tre libri di Nicolo Leonico, (16)r.

3 Venezia, Biblioteca Marciana, It. X, 73 (7097), 198r: «Come il quondam Reverendissimo Monsignore Giovanni Battista Castrodardo Canonico Bellunese, che fu uno de miei maggiori, commentò la Cantica di Dante, ritrovandomi havere, come herede in mano, fra molte erudite compositioni di prosa, e verso, anco i fragmenti de' commentari 
La tercera obra es L'Alcorano di Macometto de 1547, mientras que la cuarta en orden cronológico es una crónica de los obispos de Belluno posterior al 1548, utilizada como fuente por el conciudadano Giorgio Piloni en su Historia della città di Belluno (Venecia, 1607), pero desconocida para los historiadores locales de los siglos XVIII y XIX ${ }^{4}$.

Sin embargo, ahora es posible leer un fragmento de esta crónica en el manuscrito misceláneo F. $65^{\text {a }}$ de la Sächsischen Landesbibliothek Staats-und Universitätsbibliothek de Dresde (SLUB). Se trata del relato de la muerte del obispo Manfredi de Collalto (1321), dedicado a Giambattista II Conde de Collalto (1514-post 1570) y antiquarum rerum studiosissimus según el juicio expresado por el médico y letrado Bartolomeo Burchelati de Treviso (1548-1632) en sus Epitaphiorum dialogi septem. En la epístola dedicatoria, redactada el 8 de agosto 1568, Castrodardo nos dice que empezó a componer la crónica de los obispos de Belluno quince años antes, es decir, alrededor de 1553, subrayando además su vivo interés, compartido por su noble protector, por las obras de historia ${ }^{5}$.

De las cuatro obras mayores y conocidas del canónigo tres son obras de historia: la traducción de Leonico Tomeo, la crónica de los obispos de Belluno y la enciclopedia de L'Alcorano. Este último debe ser considerado como el volumen árabe y turco de un pequeño pero exitoso catálogo editorial de historie que incluye obras de historia antigua, de historia contemporánea europea, del Nuevo Mundo y de historiografía, para muchas de las cuales el editor, con un interés comercial e intelectual evidente, escribió por su propia mano o por lo menos firmó las epístolas dedicatorias. En la de L'Alcorano Arrivabene ofreció al embajador francés y a los lectores europeos no sólo la traducción italiana del Corán, sino también una historia de la religión islámica que fuese neutral y útil tanto en tiempo de guerra como en tiempo de paz ${ }^{6}$.

suoi sopra Dante»; Dooley, "L'unificazione del mercato editoriale"; Tommasino, "Giovanni Battista Castrodardo bellunese dantista e divulgatore del Corano".

${ }^{4}$ Piloni, Historia della città di Belluno.

5 Sächsischen Landesbibliothek - Staats-und Universitätsbibliothek, Dresde, F. 65a 89r; Schnorr von Carolsfeld, Katalog der Handschriften der Sächsischen Landesbibliothek, pp. 373-374; Burchelati, Epitaphiorum dialogi septem, $14 r$.

${ }^{6}$ L'Alcorano, (1)v-(4)r: «[...] fa grandemente di mestiero a tutti i signori e principi christiani haver qualche particolar notitia delle cose loro, per esser più pienamente instrutti e saper meglio come haversi da governare in ogni evento, o di pace o di guerra che possa nascere». 
El in quarto de L'Alcorano, surgido a partir de una coyuntura política y diplomática filoturca, no es solo una obra de polémica antiislámica; se presenta por lo tanto como una enciclopedia histórica divulgadora, escrita por un joven polígrafo de provincia que, intentando abrirse paso con virulencia en el mercado editorial veneciano y siguiendo los deseos de su editor, optó por dirigirse a un público más amplio y más popular que el del in folio latino impreso en Basilea. Hartmut Bobzin señaló igualmente que L'Alcorano presenta una estructura histórico-cronológica más clara y más accesible que la gran antología temática de Basilea y ahora se puede afirmar que esto se debe a las lecturas y a los intereses historiográficos de su traductor-compilador ${ }^{7}$.

\section{La introducción del Alcorano y la biografía de Muhammad}

Los libros propiamente históricos son dos: la introducción y el primer libro; la primera se basa en turcica contemporáneos y europeos, o cristianos como dice el autor, mientras que el primer libro se centra en la traducción de las Fabulae Saracenorum medievales contenidas en la enciclopedia de Bibliander: la Chronica mendosa, el De generatione Machumet y la Doctrina Machumet, es decir en los tratados históricos, biográficos y teológicos del Corpus islamolatinum, conocido ya como Collectio Toletana (Apéndice 1) ${ }^{8}$.

Una vez llegados a este punto estimo oportuno analizar con mayor profundidad su introducción. Ésta, selecciona y divulga textos sobre el Imperio otomano ya bastante conocidos en Italia como los de Bartholomaeus Georgievits (XXVIII $r$-XXXVIIIv), Giovanni Antonio Menavino (XXXIXr- XLVIIIv) y Ludovico Domenichi (XLVIIIv$\mathrm{L} v$ ), anticipando y sirviendo de modelo a la Historia universale dell'origine e imperio de' Turchi de Francesco Sansovino de 1560$1561^{9}$.

7 Bobzin, Der Koran im Zeitalter der Reformation, pp. 263-269: «Aber aus zwei anderen Gründen ist das Buch interessant. Der erste betriff die Komposition des Buches. Arrivabene hat nämlich den Inhalt der drei Teilbänd von Bilbianders Koranausgabe in überaus geschickter Weise zu einem ganz neuen Kompendium umgearbeitet».

8 Sobre la distinción contrastiva entre textos cristianos e islámicos en las biografías europeas de Muhammad véanse González Muñoz, Pseudo Pedro Pascual y Tommasino, "[Nota bibliográfica] Sobre el Pseudo Pedro Pascual", Al-Qantara, 33, 1 (2012), pp. 201-211.

9 Sansovino, Dell'historia universale. 
El primer capítulo La vera vita di Macometto, tratta dall'historie di christiani (II $r$-XIr ) y el segundo Della religione de' Turchi (XIrXXVIII $r$ ) son los capítulos más largos y más originales de la introducción. Adoptan textos internos y externos a la enciclopedia de Bibliander y como sólida base textual una fuente nunca señalada por la crítica, la Confusión de la secta mahomética de Juan Andrés (1515), traducida al italiano por el vasco Domingo de Gaztelu, secretario del embajador en Venecia don López de Soria (1537). Se trata de una obra que tuvo una importante repercusión en toda Europa y fue traducida al latín, francés, inglés, alemán y holandés ${ }^{10}$.

De los dos primeros capítulos de la introducción, el primero $L a$ vera vita di Macometto tratta dall'historie di cristiani (IIr-XIr) se ha revelado más interesante. Castrodardo propone dos veces la vida de Mahoma. En los primeros cuatro capítulos del primer libro $L a$ generatione di Macometto (1v-6r), La Natività di Macometto $(6 r-8 r)$, La Nodritura di Macometto (8r-10r), La Vita i costumi la forma la statura e l'oppinioni di Macometto (10v-13v), traduce literalmente el De generatione et nutritura eius y la Chronica mendosa medievales.

En el primer capítulo de la introducción, sin embargo, leyendo a Juan Andrés y a otras fuentes, elabora una nueva versión de la vida de Mahoma, en la cual encontramos un mi 'râŷ tejido con el léxico de Dante y una larga y novedosa oración renacentista pronunciada por el monje Sergio-Bahīrā (siriaco běhīrā «el elegido») y dirigida al profeta Muḥammad ${ }^{11}$.

\section{La oración del monje Sergio-Bahīrā al profeta Muhammad}

Esta oración nunca ha despertado el interés de los historiadores del siglo XX. El único que reconoció la naturaleza del texto fue Alessandro D'Ancona (1834-1915) que poseyó un ejemplar de L'Alcorano, actualmente conservado en la Biblioteca Universitaria de Pisa. En su famoso

10 Andrés, Confusión o confutación.

11 Sobre el papel de Sergio en la leyenda de Muhammad existe una amplia bibliografía; véanse especialmente Roggema, The Legend of Sergius Bahīrā; Roggema, "A Christian Reading of the Qur'ān"; Szilágyi, "Muhammad and the Monk"; Griffith, "Muhammad and the Monk Bahīrā". 
La leggenda di Maometto in Occidente (1889), D'Ancona resaltó la presencia de la oración inusual en un texto de naturaleza biográfica ${ }^{12}$.

Una vez identificado el autor y sus intereses, la presencia de una oración de tres hojas en una biografía renacentista de Muhammad no nos parece tan singular, más bien se podría comparar con los modelos clásicos de Livio y de Tácito o con las Historie fiorentine de Maquiavelo, en cuanto ejercicio oratorio en una obra de historia, entendida precisamente como opus oratorium maxime. Esta idea toma cuerpo si tenemos en cuenta que en los años cuarenta se empiezan a publicar los textos que codifican la oratoria en italiano, de acuerdo con la retórica clásica y humanista latina. Buenos ejemplos de ello pueden ser L'oratore di Giovanni Maria Memmo de 1545, la antología de Orazioni diverse di diversi rari ingegni impresas en Venecia en 1546 y la colección florentina de Orazioni diverse et nuove de Anton Francesco Doni de 1547, obras todas ellas contemporáneas de L'Alcorano y que anticipan las Diverse orazioni volgarmente scritte de Francesco Sansovino de 1561 que se convertirán en el modelo canónico en la segunda mitad del siglo XVI ${ }^{13}$.

$\mathrm{Si}$ en la antología de Sansovino prevalece la oratoria epideíctica y académica, la oración del monje Sergio-Bahīrāâ, máscara literaria bajo la cual se oculta el traductor e historiador Castrodardo, pertenece a la retórica civil, o mejor al género deliberativo (genus deliberativum) que busca persuadir para una actuación futura o, con las palabras de Heinrich Lausberg: «pretende un cambio de la situación, que hay que realizar pragmáticamente (es decir en el curso externo de los acontecimientos socialmente relevantes)» ${ }^{14}$.

Castrodardo, mezclando y actualizando las leyendas europeas y las nuevas biografías eruditas de Muhammad, nos presenta a Sergio

12 Pisa, Biblioteca Universitaria, D'Ancona 16.7.10; D'Ancona, “La leggenda di Maometto", 253: "Nella Vita di Macometto che precede L'Alcorano tradotto nuovamente dall'arabo in lingua italiana, Venezia, Arrivabene, 1547, è detto: "Siro chiamato Surgio"; ma poi è detto: "Sergio" ed è singolar cosa in questo scritto l'orazione cinquecentesca, che tiene tre pagine, colla quale questo monaco nestoriano persuade Maometto a fondare nuova religione».

${ }_{13}$ Memmo, L'oratore del magnifico dottore; Orazioni diverse di diversi rari ingegni, Venezia, s.e., 1546; Orazioni diverse et nuove di eccellentissimi auttori, Firenze, Doni, 1547; Diverse orazioni volgarmente scritte da molti uomini illustri de tempi nostri, Venezia, Sansovino, 1561; Folena, Nueva presentación de Lisio, Orazioni scelte del secolo XVI, XXII; Bárberi Squarotti, "Le forme dell'orazione”, p. 289.

${ }^{14}$ Lausberg, Elementos de retórica literaria, pp. 18-19. 
como monje sirio, según la Theologia Mahometis de Widmanstetter (1543), y como hereje y fugitivo nestoriano a la vez. Pero el SergioBahīrā de L'Alcorano di Macometto es sobre todo un elegante y peligroso orador, un «huomo di molto maggior loquenza che di dottrina», que a través de esta ficción política y literaria, construida con materiales extraídos de más de una fuente, trata de convencer a Muhammad para tomar el poder y derrocar al emperador de Bizancio Heraclio ${ }^{15}$.

Después de identificar el género literario con el que Castrodardo reelabora las leyendas occidentales sobre la vida de Mahoma mezcladas con las tradiciones biográficas recogidas por Widmanstetter y sobretodo por Juan Andrés en España y difundidas en Italia por Domingo de Gaztelu, y tras explicar las razones literarias de la elección del género oratorio, llegamos a un punto en el que se hace necesario analizar el estilo de la oratio dedicando una atención particular a su léxico.

\section{Análisis lingüístico de la oración}

En este caso deberemos buscar la inspiración en la retórica contemporánea, y de manera particular en el léxico político de los principales tratados de Maquiavelo, tanto en el vocabulario de El Príncipe como en el de los Discorsi sopra la prima deca di Tito Livio. Muhammad en las palabras del orador renacentista Sergio-Bahīrā tiene que tomar el poder a través de su virtù, es decir, su capacidad de entender rápidamente la situación política y aprovechar la occasione ofrecida por la fortuna. Corresponde a Muhammad reconocer la oportunidad que ofrece la fortuna, como a los príncipes que toman el poder armis propriis et virtute, «con las armas propias y el talento personal» del sexto capítulo de El Príncipe.

${ }^{15}$ L'Alcorano, 3v; D'Alverny, "Deux traductions latines", p. 105; ahora en D'Alverny, La connaissance de l'Islam; Widmanstetter, Mahometis Abdallae; Petrus i Pons, "La llegenda de Mahoma a l'Edat Mitjana"; De la Cruz Palma, "La llegenda de Mahoma a l'Edat Mitjana i al Renaixement".

Al-Qanțara XXXIII 2, 2012, pp. 271-296 ISSN 0211-3589 doi:10.3989/alqantara.2011.008 


\subsection{Fortuna, virtù, occasioni}

Así en la oratio de Sergio: «dirò tutte le occasioni che ti porge la buona tua fortuna, anzi quella di tutto il mondo, e insieme il modo di quelle abbracciare, percioché si spera che colla virtù tua s'habbia liberare non solamente l'Oriente ma gran parte del mondo, sì dalla tirannia di governatori di quello come dalla falsa religione di che è ripieno»; y luego: «Hor ecco come da un de lati tu vedi la mano che ti porge la felice tua fortuna». Además, ya en el relato introductor de la oración era la fortuna la que ofrecía a Mahoma la amistad del orador Sergio como una de sus nuove occasioni: «anzi, ogni dì appresentandosegli nuove occasioni, deliberò [Muhammad, nota del autor] dare al suo disegno spedito effetto. E mentre che così pensa, ecco che la fortuna, sempre nel male apparata, se gli offerisce Siro chiamato Surgio» ${ }^{16}$.

A partir de estos presupuestos trataré de anticipar algunos aspectos que no han sido considerados por «aquellos investigadores que al analizar el lenguaje de Maquiavelo no han ido más allá de los términos virtù y fortuna», tal y como el propio de Carlo Ginzburg (2010) valora esta aproximación llevada a cabo fundamentalmente en los países de habla inglesa. Para tal propósito recurriré a herramientas críticas como los análisis de los historiadores de la lengua y de la literatura, italianos y franceses, posteriores a los trabajos aún hoy determinantes de Fredi Chiappelli (1952-1969) en los que se forja la apreciación de Ginzburg ${ }^{17}$.

${ }^{16}$ L'Alcorano, 3r-4r-v; Véase también Rosaccio, Discorso di Gioseppe Rosaccio, p. 5: «Conoscendo adunque Maometto l'occasione, formò una nuova legge o setta, nella quale volse che tutte l'altre leggi havessero qualche parte»; Chiappelli, Studi sul linguaggio, p. 82: «Il Machiavelli si sforza di superare l'incalcolabilità dell'esistenza degli organismi politici: e la presenza di un elemento "fortuna" sventa ogni possibile calcolo. Gli è necessario allora distinguere una fase calcolabile anche negli avvenimenti portati dalla "fortuna"; e questa fase si viene tecnificando sotto il nome dell'occasione, l'occasione è il termine intermedio fra fortuna e virtù»; Chiappelli, Nuovi studi sul linguaggio, p. 32; Martelli, "Da Poliziano a Machiavelli"; Larosa, "Il tema dell'occasione tra ghiribizzi politici". Véase también Pozzi, "Machiavelli e Guicciardini”; Franceschini, Lingua e stile nelle opere in prosa; Bausi, "Lingua e stile".

${ }_{17}$ Ginzburg, "Maquiavelo", p. 14; véase también Capata, Il lessico dell'esclusione. 


\subsection{Fama, biasimo}

La virtù de Muhammad es famosa. Sergio-Bahīrā, al comienzo de la oración, llama la atención del profeta, subrayando la grande y formidabile fama de la virtù del profeta que él ganó por su nuovi tumulti (otro vocablo técnico de El Príncipe). Sergio señala también su riputazione acquistata solamente con l'armi, es decir, «su reputación adquirida por el uso de las solas armas» y sus laudabili imprese «sus loables empresas». Estos términos incrementan su significado, tanto aquí como en la retórica de Maquiavelo, si se relacionan en el mismo texto con sus antónimos: los reyes de Persia son infami y el emperador Heraclio es todo lo contrario que «loable»: él es tutto odiato e biasmato. Como ya argumentó Maria Cristina Figorilli: «Quello del biasimo è un motivo ricorrente nella produzione machiavelliana, carico di tensioni e risentimenti che si sprigionano dal complesso rapporto che si dà tra Machiavelli autore e i suoi lettori. Il "biasimo", considerato indipendentemente dalla natura "trista dell'uomo", nel linguaggio di Machiavelli è espressione come la "laude" del giudizio sociale»» ${ }^{18}$.

\subsection{Rovina, rimedio}

Otro tema es el de la rovina y del remedio. El emperador Heraclio, viciado por el ocio después de la victoria sobre los persas «lascia andare ogni cosa in ruina, talché per sua dappocaggine si è perduto l'Asia, la Soria e l'Egitto quasi». Los dos términos oscilan entre la metáfora -hay quien la llamó obsesión-aedificatoria (el estado como edificio), a la que pertenece también el término disegno referido a los proyectos políticos de Mahoma, y el lenguaje médico-fisiológico (el estado como cuerpo). Chiappelli llama la atención sobre un pasaje del sexto capítulo de El Príncipe que, como tendremos ocasión de ver más adelante, nos interesa singularmente: «Di qui nacque che tutti e' profeti armati vinsono, e li disarmati ruinorono» ${ }^{19}$.

18 Zanon, "Potenza"; Van Heck, "In margine agli scritti", p. 54: «Fin dai primi documenti di questo corpus s'incontrano numerosi concetti che diventeranno cardini della teorizzazione politica machiavelliana: fortuna, occasione, necessità, ingratitudine..., il trinomio danari-riputazione-forze (altra tematica presente in maniera quasi ossessiva nelle Consulte e Pratiche)»; Figorilli, Machiavelli moralista, p. 72. Véase también Marchand, Niccolò Machiavelli, pp. 115-116 (sobre fortuna y occasione).

${ }^{19}$ Chiappelli, Studi sul linguaggio, pp. 53-55; Fournel y Zancarini, Sur la langue du Prince, pp. 545-610 y 568-570 Ruinare/rovinare, ruina/rovina; pp. 579-582 
Para Giulio Ferroni en Maquiavelo es el ocio lo que genera la rovina inevitable: "perfino gli organismi politici più saldi e sicuri percorrono quella parabola dall'origine virtuosa all'ozio e alla corruzione, che li conduce alla fine», pero aquí Muhammad se pone en contra de la rovina como rimedio di tanti mali, es decir como medicamento para las enfermedades del Imperio. Y nos recuerda las metáforas fisiológicas en El Príncipe, capítulo III: "Así pasa en las cosas del Estado: los males que nacen en él, cuando se los descubre a tiempo, lo que sólo es dado al hombre sagaz, se los cura pronto; pero ya no tienen remedio cuando, por no haberlos advertido, se los deja crecer hasta el punto de que todo el mundo los ve» ${ }^{20}$.

\subsection{I modi}

Aún más importantes en relación a la fortuna de Muhammad en la Europa moderna son los modi «las maneras», palabra que Castrodardo/Sergio, elocuente lector de Maquiavelo, utiliza más de una vez. Por ejemplo: el modo con que abbracciare las occasioni, el sicuro modo para tomar el poder, y los due modi con que allettirsi «atraer» y acquistar "conquistar a los fieles». Aquí está el núcleo de la doctrina política y religiosa de la oración construida visiblemente con materiales del sexto capítulo de El Príncipe, De principatibus novis qui armis propriis et virtute acquiruntur, y del undécimo capítulo del primer libro de los Discursos sobre la primera década de Tito Livio, De la religion de los Romanos ${ }^{21}$.

\subsection{La religione simulata}

Reflexionando sobre Muhammad, el monje Sergio recuerda los «felici successi de' grandi signori, i quali de bassi principii con favore di simolata religione pervennero ad estremo grado». En su razonamiento, Muhammad ganó el poder, volviéndose famoso sola-

Le prince-médecin; pp. 582-587 Le prince-architecte et la question des fondements de l'Etat princier.

${ }^{20}$ Ferroni, Machiavelli, o dell'incertezza, cap. VI. "Rovina e rimedio", p. 123; Figorilli, "Machiavelli, «Odio» e «rovina»"; Machiavelli, Principe, III.

${ }^{21}$ Fournel, "Frontiere e ambiguità", pp. 82-85. 
mente con l'armi «sólo por su valor militar», como Rómulo o Tulio Hostilio. Pero el monje quiere aconsejar al profeta la manera para propagar y mantener su poder, recurriendo a otras estrategias que no sean sólo las de la guerra: «ma anchora in quel ch'io posso prestarti tal consiglio che ti dia molto maggior auumento che tu non pensi né che l'armi tue ti possino dare». Así que su consejo es el siguiente:

Ti issorto e ti consiglio che co'l mezzo della religione ti facci di tal autorità che non solamente tu sii per quella riverendo ma una Maestà anchora, come principial parte che si richiegga a' maneggi de' popoli, i quali malagevolmente si ponno contenire sanza di quella, aggiungendo arte et ingegno a riconciliarti i popoli, e ciò più facilmente ti potrà riuscire quanto meno temerai di opporti loro, il che fecero tutti gli antichi Heroi, Re e governatori da principio del Mondo, non havendo più sicuro modo di signoreggiare che il timore e spavento della loro simolata religione ${ }^{22}$.

Consejo reiterado en la conclusión de su argumento como en la peroratio final. Il timore della religione tiene que ser acompañado por la licenza del vivere y por la solutione di tutti i tributi:

[...] la divotione tua, la quale con due modi più che con altri si può acquistare che è la licenza del vivere et il timore della religione. Questa per rassettargli in una ferma opinione, e quell'altra per trargli de tanti mali e estorsioni di che sono vessati. Né è cosa che più si convegna, né più si richieda a riconciliarsi gli animi et al governo de' popoli che questa, la quale molto bene intesero quelli che diedero nuove leggi agli huomini.

Dunque, ad ogni tua opinione, è di mestiero che tu chiami l'auttorità dal cielo, alla quale non sarà alcuno così savio né così isperto che non solo le creda ma non la temi anchora, tanta è la virtù che di là su può venire. Laonde sarai sicuro che a tutti i tuoi pensieri non sarà oppositione alcuna, massimamente mostrando loro che ogni cosa fai a fine di scaricargli di tante oppressioni e mostrargli la via della libertà co'l pronuntiare la solutione di tutti $i$ tributi ${ }^{23}$.

${ }^{22}$ L'Alcorano, $4 v$. Para la simulata religione en la obras contemporáneas ya citadas, véase Procacci, Machiavelli nella cultura europea, pp. 118-119; Scarpa, "Giovan Maria Memmo", pp. 433-434; Ginzburg, "Maquiavelo", p. 25, que señala la posible fuente de Maquiavelo, los Facta et dicta memorabilia de Valerio Máximo 1.2, De simulata religione y también un antecedente del siglo XV en que Mahoma aparece al lado de otros qui cum deo fertur locutus (Zoroastro, Carondas, Hermes Trimegisto, Licurgo, Dracón, Solón, Numa, Salmoxis y Mahoma); Scala, "Bartholomeus Scala". Sobre Scala véase Canfora, Prima di Machiavelli, pp. 85-86.

${ }^{23}$ L'Alcorano, $5 r$. Véase el personaje de Mahoma en un texto contemporáneo de historia unversal, Collenuccio, Compendio delle historie, 14r-v: «[...] così con l'autorità de la religione, con la forza de la spada, con la licentia del vivere, a lor modo, e con la relevatione di tributi, si sottomise la Media, la Persia, la Siria, la Giudea, lo Egitto e l'Africa, e quasi tutte le provincie Christiane del Oriente, ampliando il nome e la legge 
Las teorías políticas de Sergio parecen el resultado de una lectura atenta de los Discorsi, más concretamente del pasaje muy conocido sobre Numa Pompilio:

Talché se si avesse a disputare a quale principe Roma fusse più obligata, o a Romolo o a Numa, credo più tosto Numa otterrebbe il primo grado: perché dove è religione facilmente si possono introdurre l'armi; e dove sono l'armi e non religione, con difficultà si può introdurre quella. E si vede che a Romolo, per ordinare il Senato e per fare altri ordini civili e militari, non gli fu necessario dell'autorità di Dio, ma fu bene necessario a Numa, il quale simulò di avere dimestichezza con una Ninfa, la quale lo consigliava di quello ch'egli avesse a consigliare al popolo; e tutto nasceva perché voleva mettere ordini nuovi ed inusitati in quella città, e dubitava che la sua autorità non bastasse. E veramente mai fu alcuno ordinatore di leggi straordinarie in uno popolo che non ricorresse a Dio, perché altrimenti non sarebbero accettate: perché sono molti i beni conosciuti da uno prudente, i quali non hanno in sé ragioni evidenti da poterli persuadere a altrui. Però gli uomini savi che vogliono tòrre questa difficoltà ricorrono a $\mathrm{Dio}^{24}$.

Como sabemos, el tema de un Mahoma engañador y pseudo-profeta no es nada nuevo. Castrodardo reconstruye y renueva, en el marco de un ejercicio formal oratorio y con las modernas fórmulas políticas de Maquiavelo, lo que ya podía encontrar en la literatura medieval sobre el profeta. En la Summa totius heraesis Saracenorum de Pedro el Venerable, por ejemplo, impresa en Bibliander, leemos: «quia vi gladii non potuit, religionis velamine et divini prophetae nomine rex fieri attentavit» ${ }^{25}$.

Castrodardo ofrece a sus lectores un producto moderno; remodela la vieja acusación medieval de engaño y pseudo-profecía dirigida a Muhammad según una lectura política innovadora y una retórica oratoria culta y basada, tanto en la lectura de Maquiavelo, como en un estilo oratorio elevado. Los latinismos son literarios más que de cancillería (formidabile «temible», irritare «incitar», pervenne a de un latino perve-

e la reputatione de Sarraceni». El texto fue impreso también antes por Tramezino, 1539 y 1541. Castordardo, como sabemos, trabajó por Tramezino en 1544.

${ }^{24}$ Machiavelli, Discorsi sopra la prima deca di Tito Livio, I, XI.

${ }^{25}$ Agradezco a Michelina Di Cesare por indicarme los ejemplos siguientes Speculum Historiale, liber 23, cap. 40, 41: «[...] praesertim cum paucos adhuc fautores haberet, et arte et ingenio magno usus est, ut qui Rex esse non poterat, prophetam se Dei simularet esse, et nuncium. Hoc displicuit omni parentelae et generationi eius quae vocabatur Chorais»; Alfonsi, Dialogus adversus Iudaeos, tit. V: «Certus enim es mihi non incompertum esse quis Mahometh fuerit, quomodo callida simulatione prophetam se confinxerit»; véase Di Cesare, The Pseudo-Historical Image. 
nit ad «llegó a») y la figuras son las típicas del género, como las repeticiones o recurrencias asindéticas y anafóricas, propias del movēre latino: «ogni cosa è tirannia, ogni cosa è rubberia, ogni cosa è violentia», o como: «Ad altro non studia che a raccor gemme, ad altro non attende che a compiacere meretrici e garzoni, né ad altro pensa che a caccie», o también: «Nissuno può haver udienza da lui, nissuno è più udito».

Que para Maquiavelo la religión, o mejor dicho el metus Dei, sea sólo un instrumentum regni neutral, técnico y amoral, una «impostura» como dirán en el siglo XVII o en contra una religio civil en el sentido etimológico, es decir un vínculo social fuerte y fundacional del estado moderno, es algo que todavía se pone en tela de juicio ${ }^{26}$.

Sin embargo, lo que me interesa poner de manifiesto aquí es que en un texto considerado principalmente como una traducción de fuentes latinas medievales, o más bien una paráfrasis mal hecha del texto latino de Robert de Ketton, haya un nuevo y complejo retrato de Muhammad, basado en las teorías políticas renacentistas (el profeta armado de Maquiavelo) y, tanto en el texto como en el frontispicio, en los modelos romanos antiguos ${ }^{27}$.

\section{Muḥammad, el profeta armado}

Muhammad, si no quiere ruinar ne'sua ordini nuovi como el Girolamo Savonarola del sexto capítulo de El Príncipe, tiene que basar su poder tanto en la religión como en la fuerza, llevando el libro sagrado en una mano y empuñando la espada con la otra. Éste debe tomar como modelo a Rómulo y a Numa Pompilio, o como escribirá explícitamente el jesuita Antonio Possevino (1533-1611), tanto a Numa Pompilio como a Tulio Hostilio. Possevino, en el capítulo Linguae Arabicae necessitas ad Turcas iuvandos de la Bibliotheca selecta (lib. IX, cap. IX), en el que recomienda los tratados de controversia anti-islámica para los misioneros, describe al profeta del islam, olvidando la retórica de los tratados aconsejados (Cusano, Ricoldo, etc.) y preferiéndolo en lugar de un realismo político moderno basado en el modelo mitico-histórico de los reyes romanos:

${ }^{26}$ Viroli, Il Dio di Machiavelli. Para una crítica a esta tesis, véase. Barbuto, "Lettere non tanto chiare", señalado por Ginzburg, "Maquiavelo", p. 24.

${ }^{27}$ Najemy, Machiavelli between East and West, p. 136, pone de relieve los modelos orientales de Maquiavelo.

Al-Qantara XXXIII 2, 2012, pp. 271-296 ISSN 0211-3589 doi:10.3989/alqantara.2011.008 
Numa, conficto congressu cum Dea Aegeria, populum continuit, Tullus vim omnem posuit in armis, utrumque Mahometus expressit, nam primo novas leges, quas stupidis ea tempestate Arabibus imposuit, ad Gabrielem Angelum referebat, quo monitore sese omnia mentiebatur gerere. Deinde postquam fraus in suspicione venit, praesertim ob Iudaeos circa Mecham incolentes, quibus odorandi nasus non deerat, robur induit Tullanum, et quos arte capere non poterat, viribus in novum dogma subiugavit $[\ldots]^{28}$.

En la segunda mitad del siglo XVI no sólo se describe el Imperio otomano de acuerdo con los modelos romanos y con Maquiavelo como ya demonstró Luca D'Ascia, sino también el profeta mismo del islam. Este es el camino que lleva al Mahoma del Atheismus triumphatus de Campanella, de Gabriel Naudé y después al de George Sale ${ }^{29}$.

Sin embargo, si consideramos el Mahoma de esta oración sólo como un posible antecedente del impostor o del legislador de los libertinos europeos, o bien buscando mecánicamente las fuentes medievales de su simulatio, corremos el riesgo de perder de vista un posible mensaje político, insertado en un ejercicio oratorio aparentemente inocente, y dirigido a sus lectores contemporáneos; en particular a los diplomáticos franceses y a las fuerzas antiimperiales italianas y extranjeras establecidas en Venecia en los años cuarenta ${ }^{30}$.

28 Possevino, Antonii Possevini, p. 443.

${ }^{29}$ D'Ascia, "L'impero machiavellico", pp. 111-112 y 114 (sobre el uso político de la religión); Malcolm, "The Crescent and the City of the Sun"; Prosperi, "Il principe, il cardinale, il papa", pp. 258-259: «Si è discusso se le deduzioni scettiche, libertine e atee fossero già mature nella mente di Machiavelli e celate o dissimulate perché il contesto storico non permetteva tanto ardire. La questione è stata mal posta: le tracce di sguardi acuti e disincantati sugli usi della religione sono così tante in quell'epoca che non vale per loro la categoria del "precursore" (se mai questa categoria ha avuto un qualche valore)»; véase también Vasoli, "Machiavelli, gli antichi".

${ }^{30}$ Smith, "Opium of the people"; Silk, "Numa Pompilius", pp. 877-878: «In Bartolomé de Las Casas's Apologética historia [...] Numa serves as the template fro Pachacuti, the Inca leader responsible for recontituting ancient Andean religion». Hobbes (Leviathan, I, 12) pondrá Pachacuti a lado de Mahoma, véase Stroumsa, Enlightenment Perceptions, p. 196: «While the figure of Muhammad had traditionally been that of a false prophet in the European consciousness, things were beginning to change, and he was also perceived as héros civilisateur of the Arab tribes, hence, as an Arab Numa of sorts». Como hemos visto esta idea nació muchos antes. Véase también Elmarsafy, The Enlightenment Qur'an, x: "This identification is increasingly focused on Muhammad himself, who starts to be seen as a great man and a wise legislator rather than the wicked voluptuary of medieval legend [esto en el siglo XVIII según el autor]»; Naudé, Considérations politiques, pp. 164-165; Bianchi, "La forza della religione", pp. 132-135; Khayati, Le statut de l'Islam. 
Una lectura anti-imperial y filoturca de esta oración -pronunciada en un momento político que corresponde a las últimas esperanzas de los fuoriusciti antes de la batalla de Müllberg del 24 de abril 1547-a través de la cual interpretar al Emperador Heraclio como la máscara de Carlos V y a Mahoma como Solimán el Magnífico, tal vez es un tanto aventurada. Asumir este riesgo nos permitiría comprender todas las referencias textuales a los refugiados religionis causa, a las persecuciones de los judíos, a los herejes y a la avaricia del Emperador, locus communis de las oraciones italianas antiimperiales de la época (Apéndice 2). Esta interpretación devolvería su valor a un texto de tres hojas, cuidadosamente ocultado en los textos liminares de L'Alcorano. Para comprobar todo esto, será necesario profundizar en el estudio de su autor, el desconocido Giovanni Battista Castrodardo de Belluno, comentarista de Dante y lector de Maquiavelo.

\section{Apéndice 1}

L'Alcorano di Macometto, nel qual si contiene la dottrina, la vita, i costumi, et le leggi sue. Tradotto nuovamente dall'Arabo in lingua Italiana, Con Gratie, e Privilegii, MDXLVII.

[5], L, 100, $4^{\circ}$.

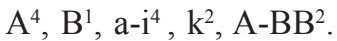

\section{Paratexto:}

Epistola dedicatoria:

Allo Illustrissimo Signor Gabriele de Luoes S. d'Aramon, consegliere della Maestà Christianissima et ambasciatore appresso il Signor Turco (firmada por Andrea Arrivabene), [2]r-[5]v.

Soneto:

Com'il gran Re del ciel Gabriello elesse (anónimo, pero de Paolo Crivelli), [5]

Epistola a los lectores piadosos:

Alli pii lettori, Ir-v.

Al-Qantara XXXIII 2, 2012, pp. 271-296 ISSN 0211-3589 doi:10.3989/alqantara.2011.008 


\section{Introducción:}

La vera vita di Macometto, tratta dall'historie di christiani, IIr-XIr.

Della religione de' Turchi, XIr-XXVIIIr.

Il matrimonio de' Turchi, XXVIIIr-XXXIIIr.

L'afflittione de Christiani schiavi, XXXIIIr-XXXVIv.

La condittione de Christiani ne' Paesi de Turchi, XXXVIIr-XXXVIIIv.

Della legge Mahomet. In che è fondata la legge Mahometana, XXXIXr.

De comandamenti della legge de' Turchi, XXXIXr-v.

La espositione del primo comandamento, che è dello amare Iddio, XXXIXv.

La espositione del secondo comandamento, dello ubidire al padre e alla madre, XXXIXv-XLr.

La espositione del terzo comandamento, del non fare altrui quello che a noi non vorremmo che fusse fatto, XLr-v.

La espositione del quarto comandamento, dello andare ai Tempij, XLv.

Come i Turchi fanno oratione nelle loro Meschit, cioè Tempij, XLIr.

Di quegli, a quali non è lecito andare alle meschite, XLI $r$-v.

La espositione del quinto comandamento, del digiuno, XLIv-XLIIr .

La espositione del sesto comandamento, del sacrificio, XLIIr-v.

Della elemosina, XLII v-XLIIIr .

La espositione del settimo comandamento che è del matrimonio, XLIII $r$ - $v$.

Del Matrimonio, che usano al presente in Turchia, XLIIIv-XLIIIIr.

De' giochi, che fanno quando la sposa esce dalla stufa, XLIIIIr.

Come va la sposa a casa del marito, XLIIIIv- XLIIIIIr.

La espositione dell'octavo, e ultimo comandamento, XLIIIIIr.

Del peccato della Superbia, XLIIIIIv.

Del peccato della Avaritia, XLIIIIIv-XLVIr.

Del vitio della Lussuria, XLVIr-v.

Del peccato della Ira, XLVIIr.

Del peccato della Gola, XLVIIr-v.

Del peccato della Invidia, XLVIIv-XLVIIIr.

Del peccato della Accidia, XLVIIIr-v.

Prophetia de Turchi, XLVIIIv-Lv.

\section{Libro primero:}

Il primo libro dell'Alcorano dove dimostrasi con qual pessime arti hebbe principio l'Imperio e la Religione di Macometto

Le cose da Dio create, $1 r-v$.

La generatione di Macometto, $1 v-6 r$. 
La Natività di Macometto, $6 r-8 r$.

La Nodritura di Macometto, 8r-10r.

La Vita i costumi la forma la statura, e l'oppinioni di Macometto, 10v-13v.

Di Eubocara successore di Macometto, $13 v-14 r$.

Aomar successore di Eubocara, 14r-v.

Odmen successore d'Aomar, 14v-15r.

Ali successore di Odmen, 15r-16r.

Alhacen successore di Ali, $16 r$.

Moauui successore di Alacen, 16v-17r.

Iezid successore di Moauui, 17r-v.

La Dottrina di Macometto, $17 v-24 v$.

\section{Libro segundo:}

Il secondo libro dell'Alcorano nel quale si contiene la legge de' Sarracini impostagli da Macometto.

Capítulos I-XXXVIII (traducción del Corán a partir de la versión latina de Roberto de Ketton), 25r-61v.

\section{Libro tercero:}

Il terzo libro dell'Alcorano nel quale medesimamente si contiene la legge di Macometto.

Capítulos I-XCVI (traducción del Corán a partir de la versión latina de Roberto de Ketton), 62r-100v.

\section{Apéndice 2}

La vera vita di Macometto, tratta dall'historie di christiani, IIIv-Vv:

Le qual cose veggendo egli [Muhammad, nota del autor] ne' suoi pensieri alcuna cosa opporsi, anzi ogni dì appresentandosegli nuove occasioni, deliberò dare al suo disegno spedito effetto. E mentre che così pensa ecco che la fortuna, sempre nel male apparata, se gli offerisce Siro chiamato Surgio, perché era come dice Philone monacho cacciato per l'heresia Nestoriana della quale era settatore dal grembo della chiesa di Costantinopoli, il quale gli aggiunse maggior copia di legna all'infiammato animo di signoreggiare. Era costui huomo di molto maggior loquenza che di dottrina, invidioso, astutissimo e bramoso d'honore come il più degli heretici sono. 
Il quale, havendolo perduto con sue false opinioni appresso Catholici Christiani, cercava, non havendo virtù né merito veruno, con Idolatri e infedeli come meglio poteva ricovrarlo.

Laonde avendo inteso di Macometto, di cui la fama era in quelle parti non solo grande ma formidabile per li nuovi tumulti che per lui erano fatti, come era securissimo ricetto de sgratiati, o qualunque altro che dalla sua rea coscientia che vi fussero riffugiti, propose andare a lui. E pensando attorno il modo che avesse tenire a farsegli grato, per haver qualche auttorità seco e insieme poterlo irritare alla vendetta di questa sua vergognosa fuga, dopo molta consideratione ministrata da Diabolico spirito gli venne in mente i felici successi de' grandi signori, i quali de bassi principii con favore di simolata religione pervennero ad estremo grado, né havendo miglior cagione fuor che questa, iscogitata come ottima introdottione, si misse in camino in Arabia, e a lunghe giornate pervenne alla città di Mecca, dove trovò Macometto in quella riputatione che di lui havea più volte inteso acquistata solamente con l'armi, al quale presentato come gli volesse dire cose nuove e grandi, fu da lui benignamente ascoltato. Così egli incominciando:

«Se grande non fusse la fama della virtù tua, o Macometto, veramente io non mi sarei mosso da luoghi ne' quali io era di riputatione, ma percioché ella anchora è di molto maggiore, che non è predicata, e che sono de' Signori nostri allettato da quella mi son ricorso alla presentia tua, e con tal disio ch'io penso più presto esser' spento dal voler di Dio che dalla voglia mia propria, come colui che suscitò egli per riffugio de' poveri oppressi di tutto il mondo, per ristoro degli afflitti dalla tirannia de' prencipi christiani, e per ridotto finalmente di tutti i cacciati dall'avaritia loro.

Laonde avisai per le persecutioni ch'io presso loro ho patito ricovrarmi appresso di te, e con fermo proponimento, veggendo come per voler divino tanto auumento di buona ventura, non solamente confortarti a seguir le laudabili tue imprese, ma anchora in quel ch'io posso prestarti tal consiglio che ti dia molto maggior auumento che tu non pensi né che l'armi tue ti possino dare, percioché intendo quando che in piacer ti sia d'esserti fedel consigliero, e in tutte le tue imprese leal coaiutore come quello che vorrei che alla voglia tua fusse governato tutto l'Oriente, il che sarebbe grandissimo contento di tutti quei popoli, per la somma giustitia di che tu sei adornato, e altre virtù o le quali risplendi. Né ad altro qui venni salvo che accenderti di giusto odio contro i signori nostri, e insieme mostrarti a castigo di tanti lor mali la via della loro perdizione come flagello da Dio mandato.

E per dar principio a miei ricordi dopo ch'io vedo l'orecchie tue attentissime alle mie parole e l'animo tuo piegato ad essequirle, dirò tutte le occasioni che ti porge la buona tua fortuna, anzi quella di tutto il Mondo, 
e insieme il modo di quelle abbracciare, percioché si spera che colla virtù tua s'habbia liberare non solamente l'Oriente ma gran parte del mondo, sì dalla tirannia di governatori di quello come dalla falsa religione di che è ripieno. Percioché non dirò de' paesi, ma nella città istessa di Heraclio ci sono cento sorti di heresia. Il nome suo il qual già pochi anni non solo era grato a tutta l'Asia, ma formidabile anchora, hora non si può sopportare da orecchia alcuna, che da per tutto è odiato e biasmato oltre modo per l'avaritia, il quale non contento più de' ordinari suoi tributi vi aggiunge taglioni a taglioni, carichi a carichi.

Più non può dire alcuno questo è mio, perché per negligentia sua il tutto è in arbitrio de' governatori. Più non ponno i miseri contadini lavorar i terreni che loro è tolto per forza le bestie di sotto il giogo e cacciati dalle proprie e paterne lor case e date a soldati, sono astretti a mille angherie di vetture e pagamenti. E per non dir più, ogni cosa è tirannia, ogni cosa è rubberia, ogni cosa è violentia, carestia e oppressione de' poveri. Cagione l'avaritia del nostro Imperadore il quale, accecato da quelle, permette queste e molto peggior cose, né d'altro ha sete se non d'oro. E insomma sono sì fattamente premuti, che più tosto bramano la morte, che menar la vita in tanti affanni.

Nissuno può haver udienza da lui, nissuno è più udito, il qual si lamenti de' suoi Thesorieri, né per alcun modo si può havere introdottione a dolersi, che con doni, e prezzo, egli è salito per quella vittoria sua nuova di Persia a tanta soperbia, e talmente impoltronito che ha lasciato a governatori, suoi assentatori tutto il maneggio dell'Imperio e la somma de' negotii, dove tutte le cose si fanno per interpreti, non si degnando di rispondere, e quando essi rispondono, dicono non quello ch'è stato loro imposto, ma quello che torna loro utile. Ad altro non studia che a raccor gemme, ad altro non attende che a compiacere meretrici e garzoni, né ad altro pensa che a caccie, crapule, giochi e diporti, e per dirlo in conchiusione dopo che egli ritornò di Persia, pare che habbia riportato insieme con la vettoria anchora quelle antiche delitie di quei Re lascivi e infami a tutto il mondo, con animo pazzo, e stordito, lasciando andare ogni cosa in ruina, talché per sua dappocaggine si è perduto l'Asia, la Soria e l'Egitto quasi.

Hor ecco come da un de lati tu vedi la mano che ti porge la felice tua fortuna. Resta mo mostrarti come habbi a pigliar l'altra e abbracciarla, con le quali medesimamente ti brama nel suo seno e ti fa di lei padrona, dove che a te altro non resta, se non che non le manchi alle cose che ella ti ministra, però io per non mancarti, ti issorto e ti consiglio che co'l mezzo della religione ti facci di tal autorità che non solamente tu sii per quella per quella riverendo ma una maestà anchora, come principial parte che si richiegga a' maneggi de'popoli, i quali malagevolmente si ponno contenire sanza di quella, aggiungendo arte e ingegno a riconciliarti i popoli. E ciò 
più facilmente ti potrà riuscire quanto meno temerai di opporti loro, il che fecero tutti gli antichi heroi, re e governatori da principio del mondo, non havendo più sicuro modo di signoreggiare che il timore e spavento della loro simolata religione.

E perché sono tanti, e di numero infinito, non starò a narrartegli come che l'ingegno tuo lo conosca o li parà ragionevole, però se mai ad alcuno si è parata miglior occasione, o paruto più commodi tempi di far questo medesimo, hora la fautrice tua fortuna a te solo lo dimostra e issorta a fare, perciocché tutte le terre de' christiani sono infettate di mille opinioni della loro fede, del che io ne son verissimo testimone come colui che cacciato della Chiesa mia mi son rifuggito a te, come rimedio di tanti mali, dove che i miseri non sanno qual parte pigliare che sia sicura all'anime loro.

Oltre poi l'Imperador istesso è tanto incrudelito con i giudei, che a loro malgrado vuole che tutti siano circoncisi, lasciandogli comunque gli piace vivere nella loro soperstitiosa religione e vana espettatione, percioché dopo la vettoria di Cosdroe vi ha aggiunto come poco ricordevole de' benefici di Dio a tante sue impietà l'arte Maga, con la quale egli è avisato che si debba guardare da gente circoncisa. I giudei dunque et i popoli christiani, scandaliggiati di tante novità di religione, sanza dubbio saranno facili ad allettirsi alla divotione tua, la quale con due modi più che con altri si può acquistare che è la licenza del vivere e il timore della religione, questa per rassettargli in una ferma opinione, e quell'altra per trargli de tanti mali e estorsioni di che sono vessati, né è cosa che più si convegna, né più si richieda a riconciliarsi gli animi, e al governo de' popoli che questa, la quale molto bene intesero quelli che diedero nuove leggi agli huomini.

Dunque ad ogni tua opinione, è di mestiero che tu chiami l'auttorità dal cielo, alla quale non sarà alcuno così savio né così isperto che non solo le creda, ma non la temi anchora, tanta è la virtù che di là su può venire. Laonde sarai sicuro che a tutti i tuoi pensieri non sarà oppositione alcuna, massimamente mostrando loro, che ogni cosa fai a fine di scaricargli di tante oppressioni e mostrargli la via della libertà co'l pronuntiare la solutione di tutti i tributi.

Veramente grande beneficio è questo, e gran mercé e grandissimo alletamento degli animi di qualunque, qualsivoglia natione, della quale tu non puoi far loro a questi tempi cosa alcuna più grata, più utile, e più desiderata, tal che le genti d'ogni paese concorreranno al favore delle tue insegne, e brameranno corcarsi nel grembo tuo, esponendo la vita, e le facoltà loro agli honori, e commodi tuoi, con che agevolmente potrai ottenire ogni cosa da te desiderata. 


\section{Bibliografía}

Andrés, J., Confusión o confutación de la secta Mahomética y del Alcorán, Ruiz García, E. (estudio preliminar), García-Monge, M. I. (trascripción del texto), Mérida, Editora Regional de Extremadura, 2003.

Bárberi Squarotti, G., Machiavelli o la scelta della letteratura, Roma, Bulzoni, 1987.

Barbuto, M. A., "Lettere non tanto chiare", La Cultura, 2 (2008), pp. 331-338.

Bausi, F., Machiavelli, Roma, Salerno, 2005.

Bianchi, L., Rinascimento e libertinismo, Studi su Gabriel Naudé, Napoli, Bibliopolis, 2006, pp. 122-136.

Bobzin, H., Der Koran im Zeitalter der Reformation. Studien zur Frühgeschichte der Arabistik und Islamkunde in Europa, Beirut-Stuttgart, In Kommission Bei Franz Steiner Verlag, 1995.

Burchelati, B., Epitaphiorum dialogi septem. Auctore Bartholomaeo Burchelato Tarvisino physico, Venetiis, ex typographia Guerraea, [1583?].

Canfora, D., Prima di Machiavelli, Politica e cultura in età umanistica, Bari, Laterza, 2005.

Capata, A., Il lessico dell'esclusione. Tipologie di Virtù in Machiavelli, Manziana (Roma), Vecchiarelli, 2008.

Chiappelli, F., Nuovi studi sul linguaggio del Machiavelli, Firenze, Le Monnier, 1969.

Chiappelli, F., Studi sul linguaggio del Machiavelli, Firenze, Le Monnier, 1952.

Collenuccio, P., Compendio delle historie del Regno di Napoli, Venezia, Per Michele Tramezino, 1548.

D’Alverny, M.T., "Deux traductions latines du Coran au Moyen Age", Archives d'histoire doctrinal et littéraire du Moyen Age, 16 (1948), pp. 69-131.

D'Alverny, M.T., La connaissance de l'Islam dans l'Occident medieval, Burnett, C. (ed.), Aldershot, Ashgate Variorum, 1994.

D'Ancona, A., "La leggenda di Maometto in Occidente", Giornale Storico della Letteratura Italiana, 13 (1889), pp. 199-281.

D'Ascia, L., "L'impero machiavellico. L'immagine della Turchia nei trattatisti italiani del Cinquecento e del primo Seicento", Quaderns d'Italià, 15 (2010), pp. 99-116.

De la Cruz Palma, Ó., “La llegenda de Mahoma a l'Edat Mitjana i al Renaixement: la compilació de Bibliander", en Història i Llegenda al Renaixement. Actes del IV Colloqui Internacional Problemes i Mètodes de Literatura Catalana Antiga. Estudi General, $23-24$ (2003-2004), Girona, Universitat de Girona-Diputació de Girona, 2004, pp. 97-112.

Di Cesare, M., The Pseudo-Historical Image of the Prophet Muhammad in Medieval Latin Literature: A Repertory, New York-Berlin, Walter De Gruyter, 2012. 
Dooley, B., "L'unificazione del mercato editoriale: i libri contabili del giornalista Apostolo Zeno", Società e storia, 14, 53 (1991), pp. 579-620.

Elmarsafy, Z., The Enlightenment Qur'an, The Politics of Translation and the Construction of Islam, Oxford, Oneworld, 2009.

Ferroni, G., Machiavelli, o dell'incertezza: la politica come arte del rimedio, Roma, Donzelli, 2003.

Figorilli, M. C., "Machiavelli, «Odio» e «rovina»: una lettura del II libro delle Istorie Fiorentine", La Rassegna della letteratura italiana, 104, 2 (2000), pp. 373-387.

Figorilli, M. C., Machiavelli moralista, Ricerche su fonti, lessico e fortuna, Napoli, Liguori, 2006.

Fournel, J. L. y Zancarini, J. C., "Sur la langue du Prince: des mots pour comprendre et agir", postfación de N. Machiavel, De principatibus-Le Prince, Paris, Presses Universitaires de France, 2000.

Fournel, J. L., "Frontiere e ambiguità nella lingua del 'Principe' condensamenti e diffusione del significato", en Pontremoli, A. (ed.), La lingua e le lingue di Machiavelli, Atti del Convegno internazionale di studi, Torino 2-4 dicembre 1999, Firenze, Olschki, 2001, pp. 71-85.

Franceschini, F., Lingua e stile nelle opere in prosa di Niccolò Machiavelli: appunti, in Cultura e scrittura di Machiavelli. Atti del Convegno di FirenzePisa, 27-30 ottobre 1997, Roma, Salerno, 1998, pp. 367-392.

Ginzburg, C., "Maquiavelo, la excepción y la regla. Líneas de una investigación en curso", Ingenium, 4 (2010), pp. 5-28.

González Muñoz, F. (ed.), Pseudo Pedro Pascual, Sobre la se[c]ta mahometana, Valencia, Universitat, 2011.

Griffith, S. H., "Muhammad and the Monk Bahīrā: Reflections on a syriac and Arabic Text from Early Abbasid Times, Oriens Christianus, 79 (1995), pp. 146-174.

Khayati, L., "Le statut de l'Islam dans la pensée du premier XVII siècle", en Heyberger, B., García Arenal, M., Colombo, E. y Vismara, P. (ed.), L'Islam visto da Occidente, Cultura e religione del Seicento europeo di fronte all'Islam. Atti del convegno internazionale, Milano, Università degli Studi, 17-18 ottobre 2007, Genova-Milano, Marietti, pp. 109-133.

Larosa, S., "Il tema dell'occasione tra ghiribizzi politici e deformazioni comiche: la lettera di Machiavelli a Luigi Guicciardini dell'8 dicembre 1509", en Machiavelli senza $i$ Medici (1498-1512). Scrittura del potere-potere della scrittura. Atti del Convegno di Losanna, 18-20 novembre 2004, Roma, Salerno, 2006, pp. 263-285.

Lausberg, H., Elementos de retórica literaria, Madrid, Gredos, 1975.

Leonico Tomeo, N., Li tre libri di Nicolo Leonico De varie historie, nuovamente tradotti in buona lingua volgare, in Venetia, [Michele Tramezino], 1544. 
Leonico Tomeo, N., Nicolai Leonici Thomaei De varia historia libri III, Venetiis, per Lucanton Iuntam, 1521.

Lisio, G., Orazioni scelte del secolo XVI, Firenze, Sansoni, 1957.

Malcolm, N., "The Crescent and the City of the Sun: Islam and the Renaissance Utopia of Tommaso Campanella", Proceedings of the British Academy, 125 (2004), pp. 41-67.

Marchand, J., Niccolò Machiavelli. I primi scritti politici (1499-1512). Nascita di un pensiero e di uno stile, Padova, Antenore, 1975.

Martelli, M., "Da Poliziano a Machiavelli sull'epigramma dell'occasione e sull'occasione", Interpres, 2 (1979), pp. 230-254.

Memmo, G. M., L'oratore del magnifico dottore et cavaliere m. Giovanmaria Memo, [In Vinetia, per Giovanni de Farri et fratelli, 1545].

Najemy, J. M, "Machiavelli between East and West", in Ramada Curto, D., Dursteler, E. R., Kirshner, J. y Trivellato, F. (ed.), From Florence to the Mediterranean and beyond, Essays in Honour of Anthony Molho, Firenze, Olschki, 2009, pp. 127-145.

Naudé, G., Considérations politiques sur le coups d'Estat, A Rome [i.e. Parigi], 1639.

Petrus i Pons, N., “La llegenda de Mahoma a l'Edat Mitjana i al renaixement: la vida del profeta", en Història $i$ Llegenda al Renaixement. Actes del IV Colloqui Internacional Problemes $i$ Mètodes de Literatura Catalana Antiga. Estudi General, 23-24 (2003-2004), Girona, Universitat-Diputació, 2004, pp. 161-172.

Piloni, G., Historia della città di Belluno, Perale M. (introducción), Bologna, Forni, 2002 [reimpresión en facsímil de la Historia di Georgio Piloni Dottor bellunese, in Venetia, MDCVII, Appresso Gio. Antonio Rampanzetto].

Possevino, A., Antonii Possevini Societatis Iesu Bibliotheca selecta qua agitur de ratione studiorum in historia, in disciplinis, in salute omnium procuranda, Romae, ex typographia Apostolica Vaticana, 1593.

Pozzi, M., "Machiavelli e Guicciardini: appunti per un capitolo di storia della prosa italiana", en Pozzi, M., Lingua e cultura del Cinquecento, Padova, Liviana, 1975.

Procacci, G., Machiavelli nella cultura europea dell'età moderna, Bari, Laterza, 1995.

Prosperi, A., "Il principe, il cardinale, il papa. Reginald Pole lettore di Machiavelli", en Cultura e scrittura di Machiavelli. Atti del Convegno di FirenzePisa, 27-30 ottobre 1997, Roma, Salerno, 1998, pp. 241-262.

Roggema, B., “A Christian Reading of the Qur'an: the Legend of SergiusBahīrā and its Use of Qur'ān and Sīra”, en Thomas, D. (ed.), Syrian Christian under Islam, The First Thousand Years, Leiden-Boston-Köln, Brill, 2001, pp. 57-74. 
Roggema, B., The Legend of Sergius Bahīrā. Eastern Christian Apologetics and Apocalyptic in Response to Islam, Leiden, Brill, 2008.

Rosaccio, G., Discorso di Gioseppe Rosaccio cosmografo all'Invitissimo Imperatore et a' Potentati Christiani, nel quale si tratta brevemente l'origine della Setta Mahometana, Firenze, per Cosimo Giunti, 1599.

Sansovino, F., Dell'historia universale dell'origine e imperio de' Turchi. Nella quale si contengono gli offici, le leggi, e i costumi di quella natione, cosi in tempo di pace, come di guerra. Con una tavola copiosissima di tutte le cose piu notabili dell'opera. Raccolta da Francesco Sansovino, in Venetia, 1560-1561 (In Venetia, appresso m. Francesco Sansovino, 1561).

Scala, B., "Bartholomeus Scala de legibus et iudiciis dialogus", Borghi, L. (ed.), La Bibliofilia, 42 (1940), pp. 256-282.

Scarpa, E., "Giovan Maria Memmo plagiario di Machiavelli”, Il Pensiero Politico, 14, 3 (1981), pp. 421-439.

Schnorr von Carolsfeld, F., Katalog der Handschriften der Sächsischen Landesbibliothek zu Dresden, Band 1. Korrigierte und verbesserte, nach dem Exemplar der Landesbibliothek photomechanisch hergestellte Ausgabe des Kataloges der Königlichen Öffentlichen Bibliothek zu Dresden, Band 1, bearbeitet von Franz Schnorr v. Carolsfeld, Dresden, Sächsische Landesbibliothek, 1979 [reimpresión facsímil de la edición Leipzig, 1882].

Silk, M., "Numa Pompilius and the Idea of Civil Religion in the West", Journal of the American Academy of Religion, 72 (2004), pp. 863-896.

Smith, M. C., "Opium of the people: Numa Pompilius in the French Renaissance”, Bibliothèque d'Humanisme et Renaissance, 52, 1 (1990), pp. 7-21.

Stroumsa, G. G., Enlightenment Perceptions of Roman Religion, in Epitomē tēs oikumenēs, Studien zur römischen Religion in Antike und Neuzeit, Cançik, H. y Cancik-Lindemaier, H. (ed.), Stuttgart, Steiner, 2002, pp. 193-202.

Szilágyi, K., "Muḥammad and the Monk: The Making of the Christian Bahīrā Legend", Jerusalem Studies in Arabic and Islam, 34 (2008), pp. 169-214.

Tommasino, P. M., "Giovanni Battista Castrodardo bellunese dantista e divulgatore del Corano (1545-1547)", Nuova Rivista di Letteratura Italiana, XIII, 1-2 (2010) [Saggi danteschi per Alfredo Stussi a cinquant'anni dalla sua laurea], pp. 343-370.

Tommasino, P. M., "Giovanni Battista Castrodardo bellunese traduttore dell'Alcorano di Macometto", Oriente Moderno, 88, 1 (2008), pp. 15-40.

Van Heck, P., "In margine agli scritti cancellereschi di Machiavelli", en Marchand, J. (ed.), Machiavelli senza i Medici, Roma, Salerno, 2006, pp. 53-71.

Vasoli, C., "Machiavelli, gli antichi, la religione e le armi", en Fontana, A., Fournel, J. L, Tabet, X. y Zancarini, J. C. (eds.), Langues et Écritures de la république et de la guerre. Études sur Machiavelli, Genova, Name, 2004, pp. 365-381.

Al-Qanțara XXXIII 2, 2012, pp. 271-296 ISSN 0211-3589 doi:10.3989/alqantara.2011.008 
Viroli, M., Il Dio di Machiavelli, e il problema morale dell'Italia, Roma-Bari, Laterza, 2005.

Widmanstetter, A., Mahometis Abdallae filii Theologia dialogo explicata, [Norimbergae], s.e., 1543.

Zanon, R., "Potenza, autorità, reputazione in Machiavelli", Cultura Neolatina, 40 (1980), pp. 319-332.

Recibido: 07/06/2011

Aceptado: 24/11/2011

Al-Qanțara XXXIII 2, 2012, pp. 271-296 ISSN 0211-3589 doi:10.3989/alqantara.2011.008 